\title{
EL SUEÑO DE LA IDENTIDAD LATINOAMERICANA O LA BÚSQUEDA DE LO PROPIO EN LO AJENO
}

\section{Héctor J. NAHuelPán MORENO*}

\section{RESUMEN}

Este trabajo relaciona la metáfora "El desierto crece (...)" con los proyectos históricos de construcción de la identidad latinoamericana. Se esboza una breve explicación sobre el significado de la metáfora enunciada por Nietzsche y luego tomada por Heidegger. Se aborda los proyectos históricos y, finalmente, se analiza cómo la búsqueda del sueño de la unidad se ha formulado de espalda a los fundamentos y la diversidad sociocultural propios del continente.

Palabras daves: Identidad latinoamericana, proyectos históricos, diversidad sociocultural.

\section{ABSTRACT}

This work relates the metaphor "the desert grows..." to the historical projects of constructing a Latin American identity. We will give a brief explanation in respect to the significance of the metaphor as enunciated by Nietzsche and later taken up by Heidegger. We will explain the historical projects and, finally, we will analyze how the search for the dream of unity has been formulated by turning its back on the foundations of sociocultural diversity characteristic of the continent.

Keywords: Latin-American identity, historical projects, socio-cultural diversity.

Recibido: 14.04.2006. Aprobado: 23.04.2007.

* Profesor Universidad de La Frontera, Temuco, Chile. Coordinador Acciones Académicas Proyecto Rüpü, Fundación Ford-Universidad de La Frontera. E-mail: hectnahu@hotmail.com / nawelpangui@gmail.com 


\section{“EL DESIERTO CRECE” Y EL RE-PENSAR LA IDENTIDAD LATINOAMERICANA}

$\mathrm{L}$

A EXPRESION "El desierto crece" constituye una metáfora enunciada por Nietzsche y posteriormente tomada por Heidegger.

[Dicha afirmación] ... en un lenguaje poético, viene a transformarse en una denuncia, en un grito de alerta (...) Ella nos impulsa a dar un salto a lo habitual, con el objeto de visualizar la angustia que encierra, de comprender la desorientación, de asumir la descripción de una situación autodestructiva, al mismo tiempo que nos permite abrir una posibilidad de reconstruir el porvenir. Sin duda, "El desierto crece (...)" forma parte de una descripción y de una expresión que es propia de la situación que vive el hombre moderno. El hombre moderno no piensa, expresa Heidegger, más bien no piensa en lo que debe pensar, cuestión ésta que constituye una situación "gravísima", en el sentido que sólo piensa la cosa, es decir, sólo se relaciona con el ente y, en ningún caso, piensa acerca del ser del ente y menos aún sobre el ser mismo (Leal, 2000: 173).

La cita anterior permite reflexionar en torno a algunos aspectos referentes a la metáfora a la cual hacemos alusión. Dicha expresión alude, en primer lugar, a una denuncia, es decir, a un llamado de atención con respecto al escenario o contexto histórico en el cual nos desenvolvemos. En cierto modo, se trata de una afirmación que pretende hacernos tomar conciencia de las contradicciones que encierra la sociedad y el sistema del cual formamos parte. Como sostiene Rubén Leal: “(...) supuestamente estamos construyendo una sociedad que dará la oportunidad de desarrollarse y de lograr la felicidad a cada uno de sus integrantes" (2000: 175). Sin embargo, diariamente los medios de comunicación informan (¿o desinforman?) respecto a la marginalidad y la exclusión social en que viven cientos de familias en el mundo y Latinoamérica, manifestaciones de violencia a causa de la delincuencia o la represión, atentados suicidas en Israel e Irak como expresión de la resistencia a los intentos "democratizadores" de Estados Unidos.

No obstante lo anterior, la expresión "el desierto crece" representa además una invitación a pensar el porvenir, "dar un salto a lo habitual", a visualizar el carácter crítico de un escenario social cuyas contradicciones se tornan destructivas.

La afirmación de Nietzsche nos habla del desierto: se refiere al lugar donde nada crece, alude al espacio donde nada vive; pero más angustiante aún, ese espacio continúa creciendo. Ese crecimiento no sólo constituye un proceso de desertificación, lo que ya es grave, sino además, nos trae la destrucción, ya que nos encontramos en una realidad devastadora, es decir, donde la destrucción es avasalladora (Leal, 2000: 174). 
Sin embargo, esta expresión nos lleva a superar un tipo de pensamiento centrado en la cosa, el ente, la forma. En suma, la expresión nos lleva a pensar en el ser, nos invita a remover aquello que se percibía como inmóvil, a desestructurar lo fijo, estable y aparentemente objetivo.

Es precisamente esta invitación a pensar en el ser, a avanzar más allá de lo aparente, lo habitual y lo inmóvil, aquello que nos permite reflexionar en torno a la identidad latinoamericana y los distintos proyectos históricos que han ido en su búsqueda desde los tiempos de la conquista hasta la actualidad. La reflexión adquiere validez si al referirnos a la identidad nos adentramos en el ámbito mismo de la esencia. Más aún cuando en la búsqueda del sueño de la unidad hemos terminado defraudándonos de nosotros mismos, al desear "llegar a ser" o "deber ser". Cuando este afán de encontrar lo propio o lo auténtico se ha realizado en lo ajeno, en integrarse a la "cultura occidental", en ser europeos o, recientemente, norteamericanos. Como ha sostenido Braulio Muñoz, "este sentido de no ser auténtico, de ser impostor, ha dejado cicatrices en el alma y en la mente" de Latinoamérica (1996: 22).

Detengámonos a continuación en los distintos proyectos tendientes a formular una identidad latinoamericana. No obstante, es necesario precisar que la construcción de la identidad se realiza a través de la historia, constituye un proceso que no se detiene en el tiempo, al contrario, está en permanente construcción y re-definición. Asimismo, la identidad constituye un estado de conciencia implícita y explícitamente compartida por unos individuos que se reconocen y expresan su pertenencia a una categoría de personas y a una comunidad que los acoge.

\section{LA BUSQUEDA DE UNA IDENTIDAD HOMOGENEA Y LOS DESAFIOS DEL PLURALISMO CULTURAL}

Según Jorge Larraín, "la constitución de una cultura latinoamericana comienza en el momento que la cultura española del siglo XVI se encuentra con las culturas indígenas de América" (1994: 33). Fue precisamente este encuentro de culturas el que, desde nuestro punto de vista, dio origen a la idea del sueño de la unidad cultural latinoamericana, en efecto, este sueño no existió antes de la llegada de los conquistadores. Los pueblos que habitaban en estos territorios estaban separados por barreras geográficas y por diferencias étnicas, culturales, económicas y políticas que, en cierto modo, hacían imposible la idea de una gran unidad o nación. Aun cuando en Mesoamérica y los Andes Centrales existieron sociedades centralizadas, como la azteca y la inca, que alcanzaron niveles de complejización sociopolíticos de importancia, coexistían grupos étnicos con diferentes idiomas e instituciones políticas, económicas y religiosas que imposibilitaban la unidad. Por ello, como ha sostenido Braulio Muñoz, "al llamar indios a los habitantes de 


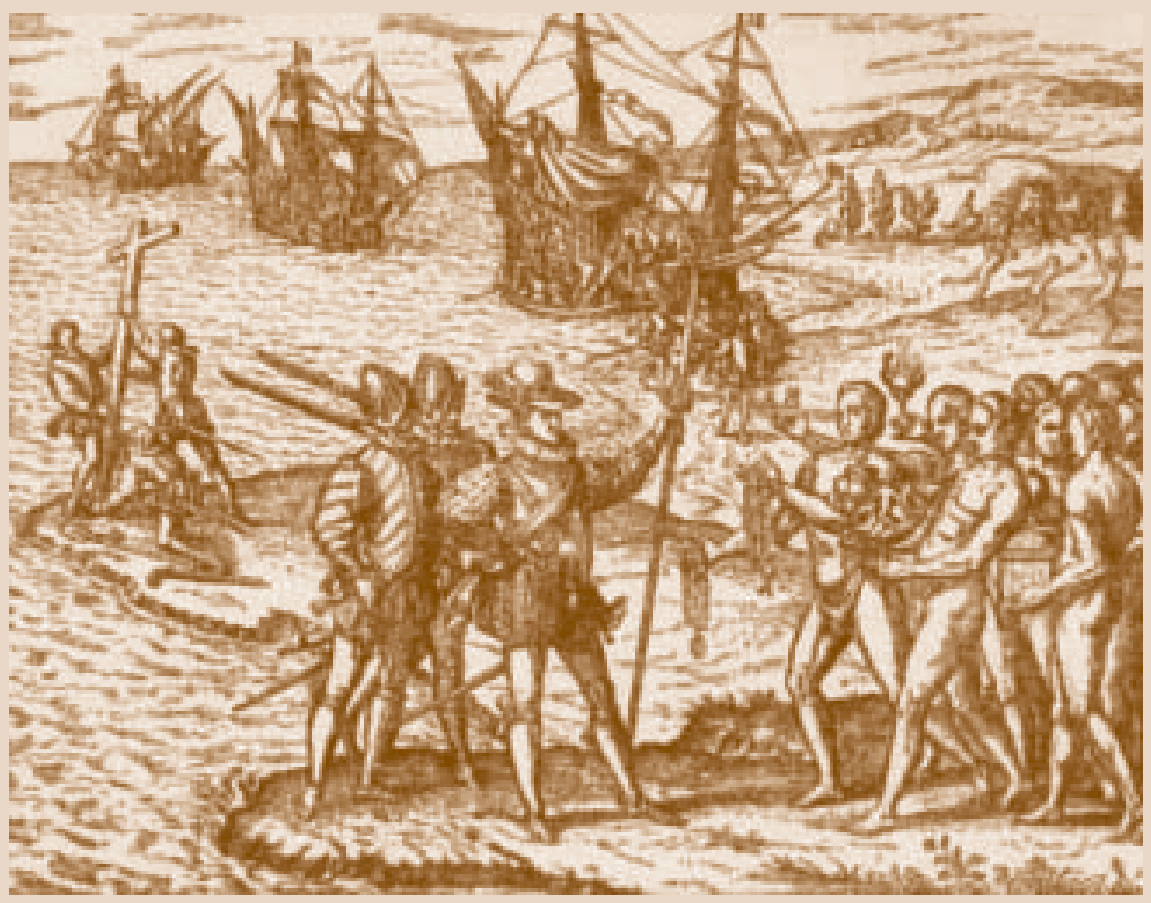

Llegada de Colón a Guanahani.

estas tierras, Colón cometió dos errores: sus anfitriones no eran súbditos hindúes y tampoco pertenecían a un solo pueblo" (1996: 17).

Sin embargo, siguiendo la idea de Muñoz, el error de Colón y la búsqueda del sueño de la unidad en la conquista, unidad representada en la hegemonía de la Corona y la religión católica, en la espada y en la cruz, derivó en la violencia y en numerosos atropellos sobre la población indígena del continente. Ataques con alevosía a poblados nativos para torturar, asesinar a varones y llevarse mujeres y niños, aquéllas para venderlas como prostitutas, estos últimos como mano de obra servil; un ejército que devino en una máquina de cazar esclavos, funcionarios y sacerdotes utilizando "peregrinos" argumentos; el Requerimiento de Palacios Rubio que afirmaba una serie de principios cristianos y el hecho de que América había sido entregada a los monarcas por el Papa. Requerimiento, éste último, que era leído en castellano a los indígenas y mediante el cual se avanzaba "con la ayuda de Dios sobre vosotros a fuego y espada".

Es cierto que la prohibición, el asesinato, la violación y el abuso de los grupos más débiles son tan antiguos como la humanidad y regían antes de la llegada del conquistador. No obstante, estas actitudes no han variado. Estados opresores siguieron abusando de los derechos más elementales, basta 
recordar el impacto que la construcción de los estados nacionales en América Latina tuvo sobre la población indígena que logró sobrevivir a la conquista. Todas ellas provocando denuncias como las del siglo XVI, tantas, por desgracia, que no es necesario mencionarlas, están en la mente de todos.

El segundo proyecto histórico de importancia en la configuración de la identidad latinoamericana habría sido la crisis de la Independencia y el nuevo modelo político y social que se intenta construir en el continente. En efecto, la gran empresa que se inaugura en el siglo XIX fue la formación del Estadonación (Pinto, 2000). Fenómeno que habría contemplado, de acuerdo a Marta Bechis, "tanto la formación y el establecimiento del nuevo Estado en sí como entidad política, como los procesos de creación de grados viables de unidad, adaptación, logro y el sentido de unidad nacional de la población" (1992: 93).

Durante el siglo XIX en América Latina, los nuevos estados y la búsqueda de una identidad en lo ajeno, se expresaron en la idea de "civilizar" que se impuso a través de la noción de progreso. Luego de la declaración de Independencia, en la mayoría de los países de América Latina, la preocupación por el progreso se configuró como base para todos los proyectos de identidad nacional. El progreso constituía el eje que debía orientar una identidad futura, una identidad "del llegar a ser" y del "deber ser" civilizados. Mientras que la barbarie fue definida como la antifigura, es decir, como un molde social opuesto al "civilizado".

En cierto modo, la construcción de la "nación política" en América Latina se realizó de espaldas a lo propio, lo mestizo, lo indígena. Al respecto, de importancia fue la influencia del positivismo y las ideas de Augusto Comte. En América Latina, para los espíritus liberales, Comte representó la secularización de la historia al cancelar el carácter providencialista de ésta y afirmar su reemplazo por una historia científica que marchaba al ritmo del progreso. Para Comte, la historia se sometía a una ley universal: la ley de los tres estados. "El primer estado era el teológico-militar, que no vacilaban en asimilar a la colonia; el segundo, el metafísico-legista: la república; y, el tercero, el que tenían que alcanzar: la sociedad científico-industrial" (Rojas, 1991: 90). Fue con ese horizonte que se formularon los proyectos de sociedad en la segunda mitad del siglo XIX en América Latina. Se trataba de un proyecto que insistía en occidentalizar e incorporar las nuevas ideas europeas de la razón y el liberalismo, más que en definir una identidad nacional. $\mathrm{Al}$ respecto, importantes fueron los planteamientos de algunos intelectuales latinoamericanos. Entre ellos Sarmiento, quien argumentaba que en América Latina existía una disputa entre civilización y barbarie. La primera venía a representar a Europa y Estados Unidos; mientras que la segunda estaba ligada al mundo indígena y a la inferioridad racial. Dicha dicotomía fue expresada en la oposición entre ciudad y campo. Al respecto, en su libro Facundo, civilización y barbarie, Sarmiento sostenía: 


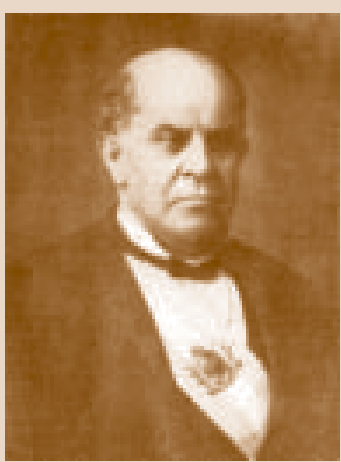

F. Sarmiento

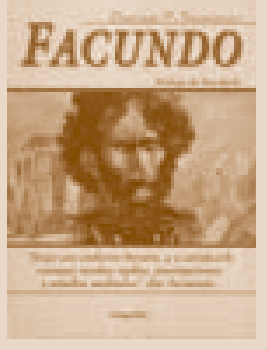

El hombre de la ciudad viste el traje europeo, vive de la vida civilizada, tal como la conocemos en todas partes: allí están las leyes, las ideas de progreso, los medios de instrucción, alguna organización municipal, el gobierno regular, etc. Saliendo del recinto de la ciudad, todo cambia de aspecto: el hombre de campo lleva otro traje, que llamaré americano, por ser común a todos los pueblos; sus hábitos de vida son diversos; sus necesidades, peculiares y limitadas; parecen dos sociedades distintas, dos pueblos extraños uno de otro (Sarmiento, 1988: 68).

De esta manera, la búsqueda de la identidad en el "progreso" y la "civilización” europea explica algunas de las políticas orientadas a modernizar América Latina durante el siglo XIX. Por ejemplo, aquellas destinadas a "mejorar la raza" mediante la inmigración europea, los avances sobre territorios indígenas que hasta entonces habían permanecido bajo una relativa autonomía, los consiguientes procesos de imposición de un arbitrario cultural "nacional” tendiente a la homogeneización de la población, la difusión de un modelo de educación científica. En suma, la construcción arbitraria de una identidad cultural y una serie de medidas orientadas a reemplazar el legado cultural colonial, mestizo e indígena.

Por otra parte, el siglo XX marcará, al menos inicialmente, un quiebre respecto a proyectos de construcción de una identidad latinoamericana anteriores. En efecto, hacia la década de 1930 y ante el impacto de la depresión económica mundial de esos años, surgen una serie de trabajos insistiendo que América Latina era diferente e incompatible con la adopción del racionalismo europeo. En este movimiento se encontraban indigenistas que abogaban por cambiar la imagen de los indígenas como sociedades atrasadas y faltas de "civilización" y llamaban a implementar reformas sociales que favorecieran a las empobrecidas comunidades indígenas (Larraín, 1994: 44). Posteriormente hacia la década de 1950, bajo la influencia de la CEPAL, se fueron promoviendo ideas relativas a la modernización e industrialización, como además el reemplazo del antiguo sistema oligárquico controlado por las viejas aristocracias terratenientes.

Luego vinieron, hacia la década del 60 y 70, las ideas sobre el imperialismo y la dependencia, ligadas al resurgimiento del marxismo, al fracaso del modelo sustitutivo de importaciones y a los esperados intentos de "independencia económica" mediante el impulso a la industrialización. Más tarde, a fines de los 70 y en los 80 ante el colapso de los proyectos socialistas, vinieron las ideas conservadoras y neoliberales de libre mercado acompañadas de dictaduras militares. En adelante, las políticas de los estados deben favorecer la inversión extranjera, abolir las tarifas aduaneras y abogar por un capitalismo dinámico que regule el bienestar social.

Actualmente, a pesar de los distintos proyectos históricos orientados a lograr unificar cultural y racialmente el continente, coexisten diferencias 
significativas. Es más, si bien hasta hace poco se pensó que la globalización tendría efectos esencialmente homogeneizadores, como consecuencia de la configuración de las relaciones de poder a nivel internacional, se visualiza un escenario en que este mismo proceso impulsa la reemergencia de culturas e identidades cuya existencia había sido negada hasta entonces. Ello se ha hecho evidente al observarse el carácter histórico limitado y por consiguiente arbitrario de las entidades nacionales o de los estados-nacionales construidos en el siglo XIX sobre la base de la negación de la pluralidad cultural (Boccara, 1999: 21). En efecto, aunque en América Latina existen algunas formas centrales de integración y síntesis, las diferencias culturales y étnicas son todavía muy importantes.

Este fenómeno que pone al descubierto la existencia de nuevas identidades y la reformulación y la adaptación sociocultural de otras, ha tenido importantes consecuencias en las ciencias sociales. Los grandes paradigmas han sido sometidos a una crítica, entre las cuales se sostiene, por ejemplo, que el marxismo menospreció el factor cultural en el estudio de las formaciones sociales (Boccara, 1999: 25).

El cuestionamiento a las categorías universales ha abierto un nuevo campo de reflexión y reformulación en la investigación social. En ellas, la identidad y la cultura pueden ser consideradas desde nuevos enfoques que contemplen el carácter dinámico de ambas. Esto último nos obliga a pensar el pluralismo cultural en Latinoamérica, a la vez que a relativizar las categorías homogeneizantes que han sostenido la existencia de una identidad continental sobre la base de esencialismos de índole indigenista, hispanista o fundamentados en la existencia de una Latinoamérica mestiza. Desde nuestro punto de vista la reflexión en torno al ser y la identidad latinoamericana no pasa por sostener la incompatibilidad entre un modelo tradicional y los procesos modernizadores impulsados hasta ahora. Más bien por re-pensar cómo ciertas formaciones culturales han ido variando en el tiempo, redefiniéndose y re-estructurándose hasta configurar un escenario cultural diverso que imposibilita homogeneizar bajo una categoría identitaria única la variedad del paisaje sociocultural del continente.

\section{REFERENCIAS}

Bechis, Marta. 1992. “Instrumentos para el estudio de las relaciones interétnicas en el periodo formativo y de consolidación de los estados nacionales". En: Hidalgo, C. y Tamagno, S. (comp.). Etnicidad eidentidad. Buenos Aires: Centro Editor de América Latina.

Boccara, Guillaume. 2000. “Antropología diacrónica. Dinámicas culturales, procesos históricos y poder político”. En: Boccara, G. y Galindo, S. (editores). Lógica mestiza en América. Temuco, Chile: Instituto de Estudios IndígenasUniversidad de La Frontera. 
Larraín, Jorge 1994. "La identidad latinoamericana. Teoría e historia”. En: Estudios Públicos, CEP, No 55, Santiago de Chile.

Leal, Rubén. 2000. "El desierto crece (...). Una denuncia al modo de pensar moderno". En: Estudios Sociales, Corporación de Promoción Universitaria, $\mathrm{N}^{\circ} 106$, Santiago de Chile.

Muñoz, Braulio. 1996. Huairapamushcas. La búsqueda de la identidad en la novela indigenista latinoamericana. Temuco, Chile: Ediciones Universidad de La Frontera.

Pinto, Jorge. 2000. Dela inclusión a la exclusión. La formación del estado, la nación y el pueblo mapuche. Santiago de Chile: IDEA (Instituto de Estudios Avanzados).

Rojas Mix, Miguel. 1991. Los cien nombres de América, eso que descubrió Colón. Barcelona: Editorial Lumen.

Sarmiento, Domingo. 1988. Facundo, civilización y barbarie. Madrid: Alianza Editorial. 